This item is the archived peer-reviewed author-version of:

\title{
Analysis and synthesis of supershaped dielectric lens antennas
}

\section{Reference:}

Bia Pietro, Caratelli Diego, Mescia Luciano, Gielis Johan.- Analysis and synthesis of supershaped dielectric lens antennas IET microwaves, antennas and propagation - ISSN 1751-8725 - 9:14(2015), p. 1497-1504

Full text (Publishers DOI): http://dx.doi.org/doi:10.1049/iet-map.2015.0091 


\section{Analysis and Synthesis of Supershaped Dielectric Lens Antennas}

\begin{tabular}{|r|l|}
\hline Journal: & IET Microwaves, Antennas \& Propagation \\
\hline Manuscript ID: & MAP-2015-0091 \\
\hline Danuscript Type: & Research Paper \\
\hline Complete List of Authors: & $\begin{array}{l}\text { Bia, Pietro; Politecnico di Bari, DEI } \\
\text { Caratelli, Diego; Antenna Company, } \\
\text { Mescia, Luciano; Politecnico di Bari, DEI - Dipartimento di Ingegneria } \\
\text { Elettrica e dell'informazione } \\
\text { Gielis, Johan; University of Antwerp, }\end{array}$ \\
\hline Keyword: & $\begin{array}{l}\text { ANTENNAS, OPTIMISATION, ELECTROMAGNETIC DEVICES, PHYSICAL } \\
\text { OPTICS, RAY TRACING }\end{array}$ \\
\hline &
\end{tabular}

SCHOLARONE ${ }^{\text {I" }}$

Manuscripts 


\title{
Analysis and Synthesis of Supershaped Dielectric Lens
}

\section{Antennas}

\section{Pietro Bia ${ }^{1}$, Diego Caratelli ${ }^{2}$, Luciano Mescia ${ }^{1}$, and Johan Gielis ${ }^{3}$}

${ }^{1}$ Dipartimento di Ingegneria Elettrica e dell'Informazione, Politecnico di Bari, Via E. Orabona 4,70125, Bari - ITALY

${ }^{2}$ The Antenna Company Nederland B.V., 2628 ZD, Delft, The Netherlands

${ }^{3}$ University of Antwerp, Groenenborgerlaan 171, 2020 Antwerp, Belgium

\section{February 3, 2015}

\begin{abstract}
A novel class of supershaped dielectric lens antennas, whose geometry is described by the three-dimensional Gielis' formula, is introduced and analyzed. To this end, a hybrid modeling approach based on geometrical and physical optics is adopted in order to efficiently analyze the multiple wave reflections occurring within the lens and to evaluate the relevant impact on the radiation properties of the antenna under analysis. The developed modeling procedure has been validated by comparison with numerical results already reported in the literature and, afterwords, applied to the electromagnetic characterization of Gielis' dielectric lens antennas with shaped radiation pattern. Furthermore, a dedicated optimization algorithm based on quantum particle swarm optimization (QPSO) has been developed for the synthesis of 3-D supershaped lens antennas with single feed, as well as with beam-forming capabilities.
\end{abstract}


Keywords - Lens antennas, Gielis' formula, high-frequency techniques, quantum particle swarm optimization.

\section{Introduction}

Dielectric lens antennas are widely used in various applications such as radar [1], millimeter wave imaging [2], radio-astronomy [3], as well as broadband wireless communications at high frequencies [4]. The attractive features of this class of antennas primarily consists in the beam collimating/shaping capability, combined with both mechanical and thermal stability, that eases the integration in densely populated electronic circuits.

In the literature, a great deal of attention has been devoted to dielectric lenses antennas with canonical (elliptical, spherical, or hyperbolic) and rotationally symmetric shapes $[5,6]$, optimized in order to enhance the directivity of the antenna and eventually the Gaussicity of the radiated beam. Few studies deal with lens antennas featuring a more complex geometry $[7,8]$. The effect of multiple internal wave reflections has been investigated only in lens antennas with classical and rotationally symmetric shapes [9], [10], [11].

The goal of this research is to present a detailed study of a new class of supershaped dielectric lens antennas whose geometry is described by the so-called Gielis' formula $[12,13]$. This formula, which generalizes the polar equation of the ellipse, allows the modeling of an extremely wide range of natural objects (plants, stems, starfish, shells, flowers, and more) alongside man-made structures, in a simple and analytical way adjusting a reduced number of parameters. This, in turn, translates into the possibility to automatically reshape the lens profile so that any automated optimization procedure could be conveniently adopted to identify the geometrical parameters yielding optimal antenna performance.

In the presented contribution, the radiation properties of the mentioned antennas are investigated by means of a dedicated high-frequency technique based on optical ray approx- 
imation [14]. In particular, geometrical optics (GO) is adopted to analyze the electromagnetic field propagation within the lens region. In doing so, the contribution of the multiple internal reflections is properly taken into account, so enhancing the accuracy of the modeling procedure especially when dielectric materials with relatively large permittivity are considered in the design. In this case, the common hypothesis that the energy content relevant to higher order reflected rays can be neglected is not applicable. Finally, by virtue of the equivalence principle and in accordance with the physical optics (PO) approach [14], the far field outside the lens can be evaluated by radiation in free space of the equivalent electric and magnetic current densities on the lens surface. In the developed methodology, these currents are calculated by applying the local Fresnel transmission coefficients along the lens surface to the electromagnetic field evaluated using GO approach. A quantum-inspired version of the Particle Swarm Optimization (PSO) algorithm, namely the Enhanced Weighted Quantum Particle Swarm Optimization (EWQPSO) has specifically developed to perform the design of the supershaped lens antennas yielding optimal antenna performance. Contrary to genetic algorithm and other heuristic techniques, PSO avoids the use of complicated evolutionary operators, is characterized by reduced computational burden and complexity, allows a more efficient global and local exploration of the search space [15]. In combination with the mentioned asymptotic modeling technique, the EWQPSO procedure allows the recovering, in a time-effective way and with reduced computational cost, of the optimal Gielis' parameters characterizing the lens shape.

\section{Antenna modeling approach}

As shown in Fig. 1, the typical antenna structure considered in this study consists of an electrically large dielectric lens placed at the center of a perfectly conducting metal disk, with radius $r_{d}$, acting as a ground plane and, at the same time, as a shield useful to reduce 


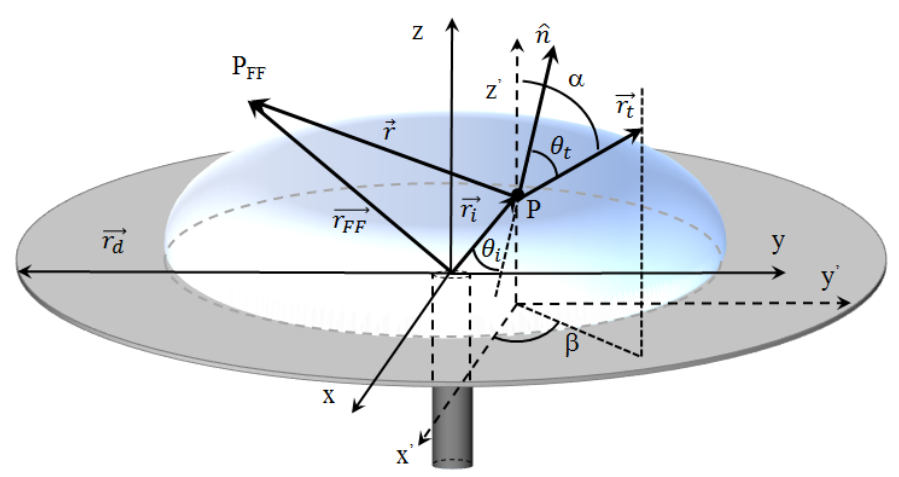

Figure 1: Geometry of a dielectric lens antenna. The reference coordinate system used to express the electromagnetic field quantities is also shown.

the back-scattered radiation. The lens is illuminated by the far-field pattern generated by a given electromagnetic source, such as open-ended waveguides, horn antennas or coaxial probes [16].

Under the assumptions of GO theory, the electric field outside the lens region can be conveniently evaluated as:

$$
\mathbf{E}_{t}=\sum_{m} \mathbf{E}_{t}^{m}
$$

where

$$
\mathbf{E}_{t}^{m}=E_{t \|}^{m} \frac{\left(\hat{\mathbf{n}} \times \hat{\mathbf{k}}_{t}^{m}\right) \times \hat{\mathbf{k}}_{t}^{m}}{\left\|\left(\hat{\mathbf{n}} \times \hat{\mathbf{k}}_{t}^{m}\right) \times \hat{\mathbf{k}}_{t}^{m}\right\|}+E_{t \perp}^{m} \frac{\hat{\mathbf{n}} \times \hat{\mathbf{k}}_{t}^{m}}{\left\|\hat{\mathbf{n}} \times \hat{\mathbf{k}}_{t}^{m}\right\|}
$$

is the transmitted electric field contribution pertaining to the $m$-th internal reflection process. In (2), $\hat{\mathbf{n}}$ denotes the unit vector normal to the lens surface, $\hat{\mathbf{k}}_{t}^{m}=\mathbf{k}_{t}^{m} /\left\|\hat{\mathbf{k}}_{t}^{m}\right\|$ is the unit vector of the transmitted wave corresponding to the $m$-th internal reflection with $\left\|\hat{\mathbf{k}}_{t}^{m}\right\|=2 \pi n_{d} / \lambda_{0}, n_{d}$ being the refractive index in the dielectric material forming the lens, $\lambda_{0}$ being the operating wavelength in the vacuum. In particular, $E_{t \|}^{m}$ and $E_{t \perp}^{m}$ are the parallel and orthogonal components, respectively, of the transmitted wave contribution due to the $m$-th reflected beam. These field quantities are determined by multiplying the parallel, $E_{i \|}^{m}$, and orthogonal, $E_{i \perp}^{m}$, components of the incident electric field, $\mathbf{E}_{i}^{m}$, on the lens surface with the proper Fresnel transmission coefficients. The evaluation of both $E_{i \|}^{m}$ and $E_{i \perp}^{m}$ is carried 
out using the following equations [7]:

$$
\begin{aligned}
& E_{i \perp}^{m}=\mathbf{E}_{i}^{m} \cdot \frac{\hat{\mathbf{n}} \times \hat{\mathbf{k}}_{i}^{m}}{\left\|\hat{\mathbf{n}} \times \hat{\mathbf{k}}_{i}^{m}\right\|} \\
& E_{i \|}^{m}=\mathbf{E}_{i}^{m} \cdot \frac{\left(\hat{\mathbf{n}} \times \hat{\mathbf{k}}_{i}^{m}\right) \times \hat{\mathbf{k}}_{i}^{m}}{\left\|\left(\hat{\mathbf{n}} \times \hat{\mathbf{k}}_{i}^{m}\right) \times \hat{\mathbf{k}}_{i}^{m}\right\|}
\end{aligned}
$$

The incident electric field $\mathbf{E}_{i}^{m}(\mathrm{P})$ at the point $\mathrm{P}$ on the lens surface is directly computed by using the far-field pattern of the source if $m=1$. On the other hand, for $m>1, \mathbf{E}_{i}^{m}\left(\mathrm{P}_{m}\right)$ is derived starting from the $m$-th reflected wave contribution as:

$$
\mathbf{E}_{i}^{m}\left(\mathrm{P}_{m}\right)=\mathbf{E}_{r}^{m-1}\left(\mathrm{P}_{m-1}\right) \mathrm{e}^{\mathrm{j} k_{i} d}
$$

where $k_{i} d$ is the electric length between the observation point $\mathrm{P}_{m}$ and the point $\mathrm{P}$ at which the reflection takes place. The reflected field $\mathbf{E}_{r}^{m-1}\left(\mathrm{P}_{m-1}\right)$ appearing in (5) is given by:

$$
\mathbf{E}_{r}^{m-1}\left(\mathrm{P}_{m-1}\right)=E_{r \|}^{m-1} \frac{\left(\hat{\mathbf{n}} \times \hat{\mathbf{k}}_{r}^{m-1}\right) \times \hat{\mathbf{k}}_{r}^{m-1}}{\left\|\left(\hat{\mathbf{n}} \times \hat{\mathbf{k}}_{r}^{m-1}\right) \times \hat{\mathbf{k}}_{r}^{m-1}\right\|}+E_{r \perp}^{m-1} \frac{\hat{\mathbf{n}} \times \hat{\mathbf{k}}_{r}^{m-1}}{\left\|\hat{\mathbf{n}} \times \hat{\mathbf{k}}_{r}^{m-1}\right\|}
$$

where the parallel, $E_{r \|}^{m-1}$, and orthogonal $E_{r \perp}^{m-1}$ components of the $(m-1)$-th reflected wave are computed by multiplying the corresponding components of the $\mathbf{E}_{i}^{m-1}$ with the proper Fresnel reflection coefficients. In (3)-(4), $\hat{\mathbf{k}}_{i}^{m}=\mathbf{k}_{i}^{m} /\left\|\mathbf{k}_{i}^{m}\right\|$ is the unit vector of the incident wave.

Once the GO electromagnetic field has been evaluated, the equivalent electric, $\mathbf{J}_{s}$, and magnetic, $\mathbf{M}_{s}$, current densities along the lens surface can be evaluated in a straightforward manner. These currents density, according to the PO formulation, allow the calculation of the electromagnetic far-field radiated by the lens antenna at the observation point $\mathrm{P}_{\mathrm{FF}}\left(r_{\mathrm{FF}}, \theta_{\mathrm{FF}}, \phi_{\mathrm{FF}}\right)$ by considering the integral expression [7]:

$$
\mathbf{E}_{\mathrm{FF}}=\mathrm{j} \frac{\mathrm{e}^{-\mathrm{j} k_{0} r_{\mathrm{FF}}}}{2 \lambda_{0} r_{\mathrm{FF}}} \int_{S}\left[\eta_{0} \mathbf{J}_{s} \times \hat{\mathbf{u}}_{0}-\mathbf{M}_{s}(\mathrm{P})\right] \times \hat{\mathbf{u}}_{0} e^{\mathrm{j} k_{0} \mathbf{r} \cdot \hat{\mathbf{u}}_{0}} \mathrm{~d} S
$$

where $S$ is the lens surface, $\eta_{0}$ is the characteristic impedance of the vacuum, $r_{\mathrm{FF}}$ is the distance between the observation point $\mathrm{P}_{\mathrm{FF}}$ and the origin of the coordinate system, $\mathbf{r}$ is the 
vector pointing from the point on the lens $\mathrm{P}$ to $\mathrm{P}_{\mathrm{FF}}$, and $\hat{\mathbf{u}}_{0}$ is the unit vector corresponding to $\mathbf{r}$. In this way, the directivity of the considered radiating system can be obtained by the following expression:

$$
h\left(\theta_{\mathrm{FF}}, \phi_{\mathrm{FF}}\right)=\frac{4 \pi r_{\mathrm{FF}}^{2}\left\|\mathbf{E}_{\mathrm{FF}}\right\|^{2}}{\eta_{0} P_{\mathrm{tot}}}
$$

with $P_{\text {tot }}$ being the total power radiated by the lens.

As an alternative to the GO-PO method, a full-GO approach has been, also, developed. In this case, the antenna far field is computed straightforwardly by enforcing the power conservation at the interface of the lens by means of the following formula [7]:

$$
h[\alpha(\theta, \phi), \beta(\theta, \phi)]=\frac{K g_{t}(\theta, \phi) \sin \phi}{\sin \alpha\left[\frac{\partial \alpha}{\partial \theta} \frac{\partial \beta}{\partial \phi}-\frac{\partial \alpha}{\partial \phi} \frac{\partial \beta}{\partial \theta}\right]}
$$

which provides the intensity of the radiated field in outgoing direction $(\alpha, \beta)$ from a given point on the lens having spherical coordinates $(\theta, \phi)$. In $(9), g_{t}(\theta, \phi)$ is the intensity of outgoing transmitted electromagnetic field outside the lens, evaluated as:

$$
g_{t}(\theta, \phi)=\left\|\mathbf{E}_{t}\right\|^{2}
$$

Finally, $K$ is the normalization constant defined as:

$$
K=\frac{\text { Far field radiated power }}{\text { Lens outgoing power }}=\frac{\int_{0}^{2 \pi} \int_{0}^{\pi / 2} h(\alpha, \beta) \mathrm{d} \alpha \mathrm{d} \beta}{\int_{0}^{2 \pi} \int_{0}^{\pi / 2} g_{t}(\theta, \phi) \sin \theta \mathrm{d} \theta \mathrm{d} \phi}
$$

The full-GO method is much faster but less accurate than the combined GO-PO method, and therefore it is just adopted for a preliminary, rough analysis of the considered radiating structure.

In the proposed study, the geometry of the lens is described by the three-dimensional extension of the so-called superformula, introduced by Gielis, in order to describe complex natural and abstract shapes in a simple and analytical way [12]. In particular, upon assuming a Cartesian coordinate system, the general Gielis' surface can be described in terms of the 
following parametric equations [17]:

$$
\begin{aligned}
& x=R(\nu) R(\mu) \cos \nu \cos \mu \\
& y=R(\nu) R(\mu) \sin \nu \cos \mu \\
& z=R(\mu) \sin \mu
\end{aligned}
$$

where

$$
\begin{aligned}
& R(\nu)=\left(\left|\frac{\cos \frac{m_{1} \nu}{4}}{a_{1}}\right|^{n_{1}}+\left|\frac{\sin \frac{m_{2} \nu}{4}}{a_{2}}\right|^{n_{2}}\right)^{-\frac{1}{b_{1}}} \\
& R(\mu)=\left(\left|\frac{\cos \frac{m_{3} \mu}{4}}{a_{3}}\right|^{n_{3}}+\left|\frac{\sin \frac{m_{4} \mu}{4}}{a_{4}}\right|^{n_{4}}\right)^{-\frac{1}{b_{2}}}
\end{aligned}
$$

In (12)-(16) $\mu \in[0, \pi / 2]$ and $\nu \in[-\pi, \pi]$ denote convenient angle parameters. Moreover, $n_{p}, m_{p}, b_{q} \in \mathbb{R}^{+}$(positive real numbers), $p=1 \ldots 4$ and $q=1 \ldots 2$ and $a_{p} \in \mathbb{R}_{0}^{+}$(strictly positive real numbers) are selected in such a way that the surface of the lens is closed and characterized, at any point, by curvature radius larger than the working wavelength, in accordance with the GO formulation. The conventional spherical angles $\theta$ and $\phi$ (see Fig.1) are obtained from (12)-(14) as:

$$
\begin{aligned}
& \theta=\cos ^{-1}\left(\frac{z}{r}\right) \\
& \phi=\tan ^{-1}\left(\frac{y}{x}\right)
\end{aligned}
$$

where $r=\sqrt{x^{2}+y^{2}+z^{2}}$.

\section{Model validation}

The developed GO-PO ray-tracing and tube-tracing procedures have been validated by comparison with the full-wave Finite Integration Technique (FIT) adopted in the commercially available electromagnetic solver CST Microwave Studio. To this end, a supershaped lens antenna with refractive index $n_{d}=1.42$ (electric permittivity $\varepsilon_{r_{d}}=2.02$ ), having a maximum radius $r_{\max }=20 \mathrm{~cm}$ and described by the following Gielis parameters $n_{1}=n_{2}=n_{3}=n_{4}=4$, 
$a_{1}=a_{2}=a_{3}=a_{4}=1, m_{1}=m_{2}=m_{3}=m_{4}=2, b_{1}=b_{2}=2$, has been analyzed. This radiating structure has been optimized at frequency $f=10 \mathrm{GHz}$. In particular, to reduce the back radiation level a metal disk with radius $r_{p}=40 \mathrm{~cm}$ is used as a ground plane. The antenna feeding is performed using a circular waveguide, having a diameter $a=4 \mathrm{~cm}$ and filled up with the same dielectric material forming the lens. In addition, to enhance the modeling accuracy, multiple reflection contributions $(m=3)$ have been taken into account. In Fig. 2, the comparison between the normalized directivity of the antenna as computed by the developed asymptotic techniques and the full-wave analysis based on FIT approach, is shown. As it can be noticed, a closer agreement with the full--wave results is achieved by using the GO-PO tube-tracing procedure. However, it is important to stress that the rigorous full-wave analysis is by far more demanding in terms of memory occupation and simulation time as the size of the dielectric lens increases. As a matter of fact, using a workstation with dual Intel Xeon E5645 processor, frequency of $2.4 \mathrm{GHz}$, the computational time and memory allocation required by the full-wave solver are about $17400 \mathrm{~s}$ and 10.3 GByte, respectively. On the other hand, the developed design procedure is characterized by a computational time and memory allocation of $6240 \mathrm{~s}$ and 2.5 GByte, on the same workstation. The usefulness of the proposed approach is apparent especially when antenna synthesis, typically entailing a large number of optimization steps, is performed.

\section{Synthesis of lens antenna}

The Gielis superformula provides the possibility of automatically reshaping the lens profile by acting on a reduced number of parameters. As result, any automated optimization procedure can be conveniently adopted in order to identify the lens parameters yielding optimal antenna characteristic. To this aim, a dedicated novel synthesis procedure based on an improved version of the Weighted Quantum Particle Swarm Optimization (WQPSO), 


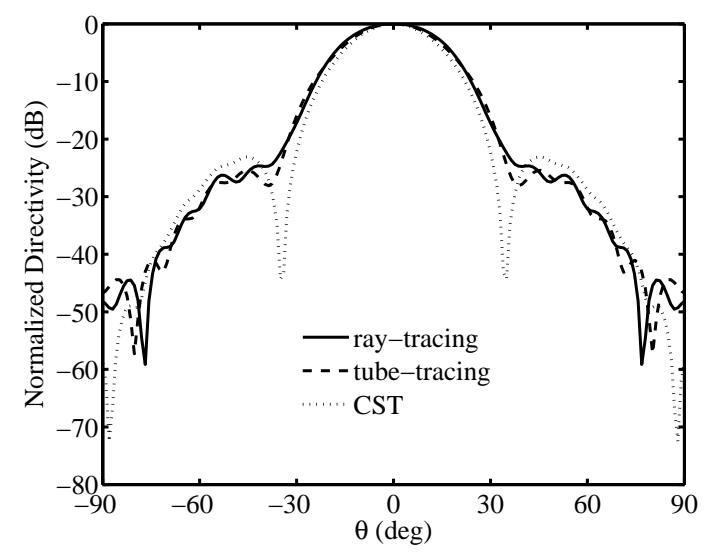

(a)

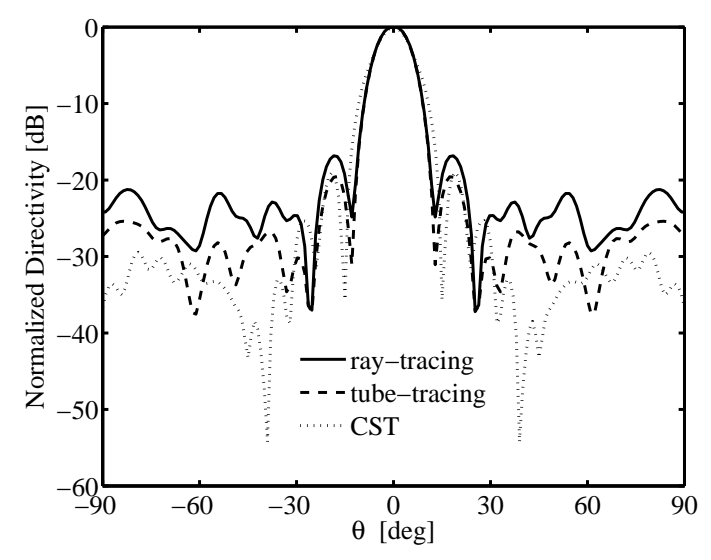

(b)

Figure 2: a) H-plane and b) E-plane radiation pattern of a supershaped lens as evaluated by GOPO ray-tracing, GO-PO tube-tracing techniques, and the finite integration technique implemented in the full-wave solver CST Microwave Studio. 
has been developed. We refer to the proposed enhanced optimization method as EWQPSO. Contrary to the conventional PSO algorithm, the considered quantum-inspired version of the PSO permits all particles to have a quantum behavior instead of the classical Newtonian one. In this way, superior performance in terms of reduced computational times can be achieved especially when a large number of parameters have to be optimized. Moreover, the EWQPSO is easier to implement since no velocity vectors for particles are needed and, therefore, a smaller set of parameters has to be dealt with.

In the conventional WQPSO presented in [18], each particles is located not just in a fixed location, $\mathbf{x}_{j}=\left[x_{j 1}, x_{j 2} \ldots, x_{j N}\right]^{T}$, but probabilistically in complete $N$-dimension search space according to a probability density function (pdf) obtained by solving the following Schrodinger equation:

$$
\mathrm{j} \hbar \frac{\partial Q\left(\mathbf{r}_{j}, t\right)}{\partial t}=\hat{H}\left(\mathbf{r}_{j}\right) Q\left(\mathbf{r}_{j}, t\right)
$$

with $\hbar$ the Plank constant divided by $2 \pi$, and where $\hat{H}(\mathbf{r})$ denotes the time-independent Hamiltonian operator that is:

$$
\hat{H}\left(\mathbf{r}_{j}\right)=-\frac{\hbar^{2}}{2 m} \nabla^{2}+V\left(\mathbf{r}_{j}\right)
$$

where $m$ denotes the mass of the particle (in the simulations equal to 1 ), and $\mathbf{r}_{j}$ is the $N$-dimension distance vector between the evaluated position $\mathbf{x}_{j}$ of the $j$-th particle and the position $\mathbf{p}_{j}$ of the local attractor. In $(20), V\left(\mathbf{r}_{j}\right)$ is the potential function defined as a Dirac distribution centered in $\mathbf{p}_{j}$. In the following, let us denote the coordinate of the personal best location of the $j$-th particle as $\mathbf{x}_{b j}(t)$ and the global best location as $\mathbf{x}_{g}(t)$. Under these assumptions, each local attractor is positioned in:

$$
\mathbf{p}_{j}(t)=\varphi_{j} \mathbf{x}_{b j}(t)+\left(\mathrm{I}-\varphi_{j}\right) \mathbf{x}_{g}(t)
$$

with $j=1 \ldots M$, where $M$ is the number of particles, and where $\varphi_{j}$ is a $M \times N$ diagonal matrix having diagonal elements values uniformly distributed in the interval $[0,1]$. Upon 
solving (19), the pdf function $Q\left(\mathbf{r}_{j}\right)$ and the updated position $\mathbf{x}_{j}(t+1)$ of the general particles are found to be, respectively:

$$
\begin{gathered}
Q\left(\mathbf{r}_{j}, t\right)=\frac{1}{\mathbf{L}_{j}(t)} \exp \left\{-2 \frac{|\mathbf{r}|}{\mathbf{L}_{j}(t)}\right\} \\
\mathbf{x}_{j}(t+1)=\left\{\begin{array}{l}
\mathbf{p}_{j}(t)+\frac{\mathbf{L}_{j}(t)}{2} \ln \left(\frac{1}{\mathbf{u}_{j}}\right) \quad \text { with } \boldsymbol{\psi}_{j}>0 \\
\mathbf{p}_{j}(t)-\frac{\mathbf{L}_{j}(t)}{2} \ln \left(\frac{1}{\mathbf{u}_{j}}\right) \quad \text { with } \boldsymbol{\psi}_{j} \leq 0
\end{array}\right.
\end{gathered}
$$

where $\boldsymbol{\psi}_{j}$ and $\mathbf{u}_{j}$ are two $N \times 1$ column vectors having elements values uniformly distributed in the interval $[0,1]$, and $\mathbf{L}_{j}(t)$ is a $N \times 1$ column vector denoting the standard deviation of the probability density function of each particle evaluated as:

$$
\mathbf{L}_{j}(t)=2 \beta\left|\mathbf{m}(t)-\mathbf{x}_{j}(t)\right|
$$

with $\beta$ being the contraction-expansion coefficient, whose value ranges from 0.5 to 1 . Generally, $\beta$ is a function of the generation index. In the framework of the conventional WQPSO algorithm, the mean best coordinates $\mathbf{m}(t)$ are evaluated as follows:

$$
\mathbf{m}(t)=\frac{1}{M} \sum_{j=1}^{M} \alpha_{j} \mathbf{x}_{b j}(t)
$$

where $\alpha_{j}$ is a suitable weighting coefficient linearly from 1.5 , for the best particle, down to 0.5 for the worst one. The developed EWQPSO technique differs from the WQPSO prominently in the definition of $\mathbf{m}(t)$. In particular, the following adaptive generation-dependent update equation is used

$$
\mathbf{m}(t)=\frac{\sum_{j=1}^{M}\left(1-\frac{F\left(\mathbf{x}_{b j}\right)}{\max \left[F\left(\mathbf{x}_{b 1}\right) \ldots F\left(\mathbf{x}_{b M}\right)\right]}\right) \mathbf{x}_{b j}(t)}{\sum_{j=1}^{M}\left(1-\frac{F\left(\mathbf{x}_{b j}\right)}{\max \left[F\left(\mathbf{x}_{b 1}\right) \ldots F\left(\mathbf{x}_{b M}\right)\right]}\right)}
$$

Each particle is relevant to a specific parameter affecting the shape and size of the lens antenna, as well as the position of the feed and the refractive index of the material forming the lens. In (26) the fitness function value $F$ is evaluated as

$$
\begin{gathered}
F\left(\mathbf{x}_{b j}\right)=\sum_{l=1}^{N_{\theta}} \sum_{m=1}^{N_{\phi}}\left|\frac{\bar{h}_{l, m}^{T}-\bar{h}_{l, m}^{j}}{1+\bar{h}_{l, m}^{T}}\right| \\
\text { IET Review Copy Only } \\
11
\end{gathered}
$$


where $\bar{h}^{T}$ is the target normalized directivity, expressed in $\mathrm{dB}$, and $\bar{h}^{j}$ is the normalized directivity, in $\mathrm{dB}$, relevant to the $j$-th particle. In (27), $N_{\theta}$ and $N_{\phi}$ denote the number of points in which the azimuthal and polar coordinates are discretized, respectively. The optimization procedure stops when the fitness value of the global best particle is smaller than a given threshold (which in the current study has been set to $10^{-3}$ ) or, alternatively, when the maximum number of particle generations is reached. The fitness function (27) has been engineered in order to obtain reliable numerical results and a fast convergence of the antenna directivity to the target one.

In order to enhance the effectiveness of the proposed lens synthesis tool, a dynamic selection of the antenna analysis technique is performed. In particular, upon denoting the maximum number of particle generations with $g_{\max }$ and current generation with $g$, the optimization algorithm is based on three main steps. During the first step, when $1 \leq g \leq$ $g_{\max } / 6$, the full-GO technique is adopted. This methodology is computationally inexpensive and fast, although less accurate and, therefore, it allows discarding efficiently the worst antenna configurations during the firsts stages of the PSO procedure. As soon as the number of generations increases $\left(g_{\max } / 6 \leq g \leq g_{\max } / 3\right)$ and the particles tend to get closer to the optimal lens geometry (second step), the GO-PO ray tracing method is used. This technique provides a higher accuracy in the characterization of the best solution candidates, although it requires larger computational resources and time because of the numerical burden related to the evaluation of the equivalent electric and magnetic current densities. Finally, during the third step, when $g_{\max } / 3 \leq g \leq g_{\max }$, the GO-PO tube tracing method with conformal tessellation of the lens surface is applied in order to achieve a more accurate modeling of multiple internal reflections and, therefore, more rigorous characterization of the antenna solutions. Of course, the use of the GO-PO methodology results in an extra computational burden. It is to be stressed that the illustrated procedure has been adjusted heuristically by 
analyzing the features of the typical problem space for the synthesis of Gielis' lens antennas.

The validation of the developed EWQPSO optimization tool has been carried out by synthesizing a lens antenna featuring a flat-top radiation pattern at frequency $f=60 \mathrm{GHz}$ (see Fig. 3). The structure is assumed fed by a rectangular patch having dimensions $a=$ $1.2 \mathrm{~mm}$ and $b=0.9 \mathrm{~mm}$, working on the fundamental mode, and backed by a circular metal ground plane with radius $r_{p}=60 \mathrm{~mm}$ (see Fig. 1). A swarm of $M=24$ particles has been used for the optimization procedure carried out over $g_{\max }=40$ generations. The position vector is $\mathbf{x}=\left[n_{1}, n_{2}, m_{1}, m_{2}, n_{3}, n_{4}, m_{3}, m_{4}, b_{1}, b_{2}, n_{d}, r_{\min }\right]^{T}$. The multidimensional search space has been restricted by assuming that the parameters $n_{1}, n_{2}, m_{1}, m_{2}, n_{3}, n_{4}, m_{3}, m_{4}, b_{1}, b_{2}$ can range from 1 to $5, n_{d}$ can vary between $1.7\left(\varepsilon_{r_{d}} \approx 2.9\right)$ and $3\left(\varepsilon_{r_{d}} \approx 9\right)$, the minimum lens radius $r_{\min }$ is assumed to range from $20 \mathrm{~mm}$ to $50 \mathrm{~mm}$, whereas the Gielis' parameters $a_{1}, a_{2}, a_{3}, a_{4}$ are all set to 1 . The location of the feeding patch is $x=y=0$ (see Fig. 1 ). Under these assumptions, by using the EWQPSO procedure, the optimal lens parameters are found to be: $n_{1}=2.480, n_{2}=2.297, m_{1}=1.503, m_{2}=1.609, n_{3}=2.986, n_{4}=1.779$, $m_{3}=1.948, m_{4}=2.845, b_{1}=4.638, b_{2}=4.888, n_{d}=1.8\left(\varepsilon_{r_{d}} \approx 3.2\right), r_{\min }=27.9 \mathrm{~mm}$ corresponding to $r_{\max }=29.8 \mathrm{~mm}$. The resulting lens geometry is shown in Fig. 4 . As it appears from Fig. 3, the synthesized radiation patterns are in excellent agreement with the target flat-top masks along both the E and H planes of the antenna. Moreover, it is clear that accounting for the multiple wave reflections occurring within the lens is instrumental to the enhancement of the modeling accuracy of the procedure. Fig. 5 shows the convergence rate of the new optimization procedure (EWQPSO), the WQPSO and the classical PSO when applied to the synthesis of the lens antenna illustrated in Fig. 4. Clearly, the EWQPSO outperforms both the PSO and the conventional WQPSO for a given number of iterations, accuracy and population size. Moreover, it is apparent that to obtain better accuracy the EWQPSO converges faster than the aforementioned alternative techniques. 


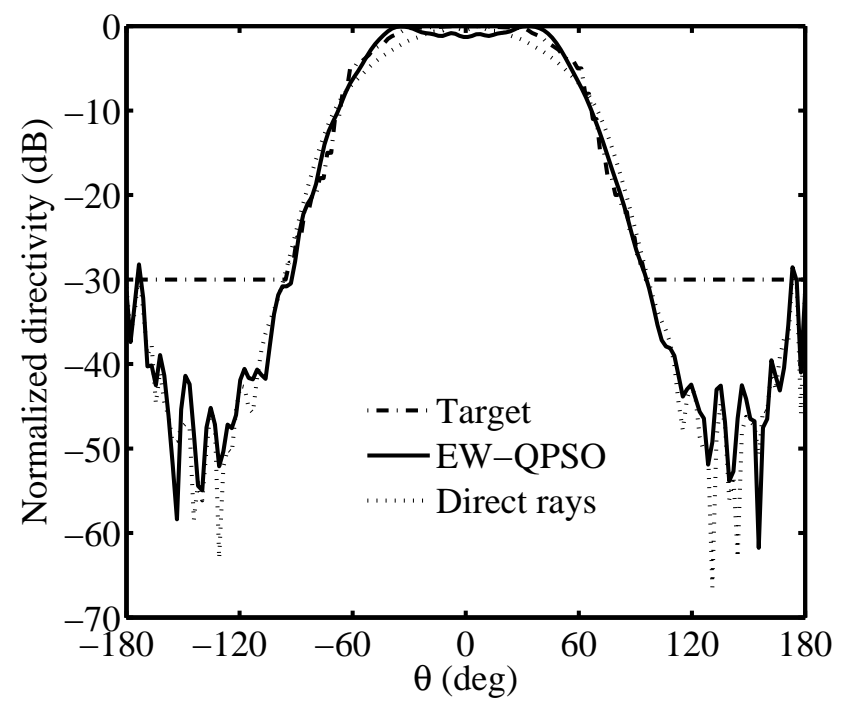

(a)

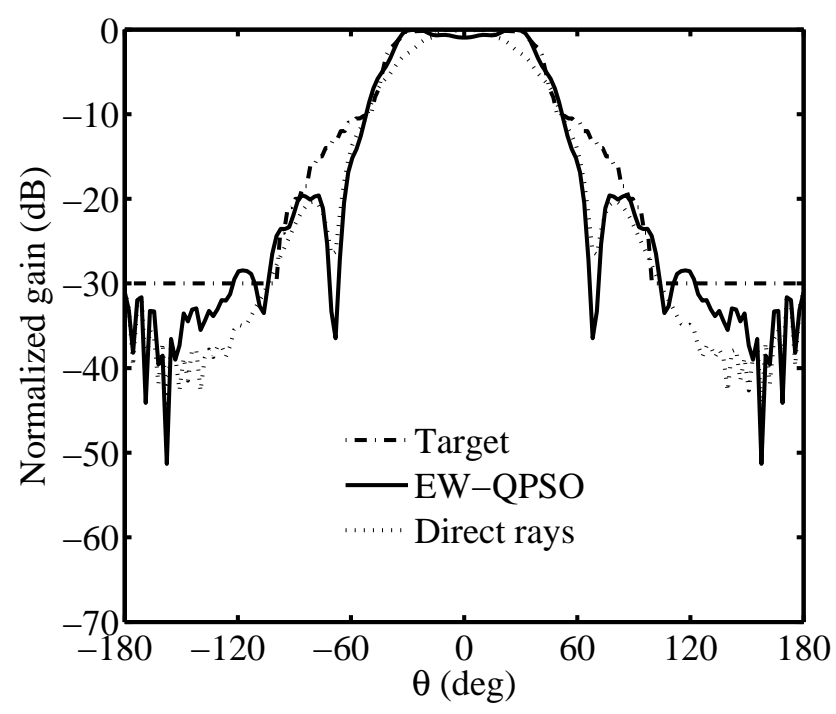

(b)

Figure 3: a) H-plane and b) E-plane radiation pattern of a Gielis' lens antenna synthesized by means of the EWQPSO procedure. The target flat-top mask and simpler direct rays results are also shown. 


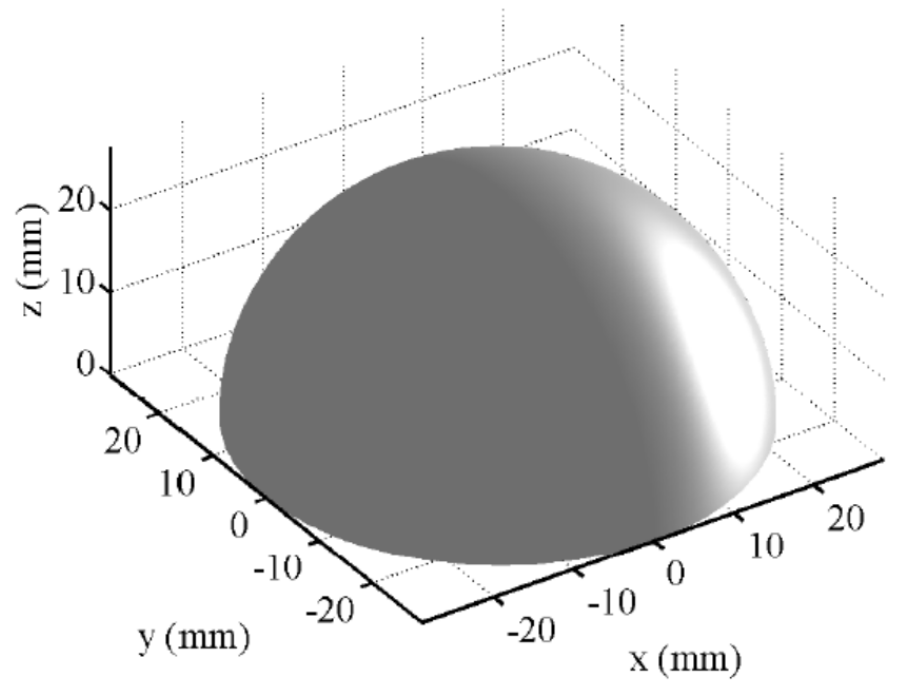

Figure 4: Shape of the lens synthesized by means of the EWQPSO procedure in order to ensure a flat-top radiation pattern at $60 \mathrm{GHz}$.

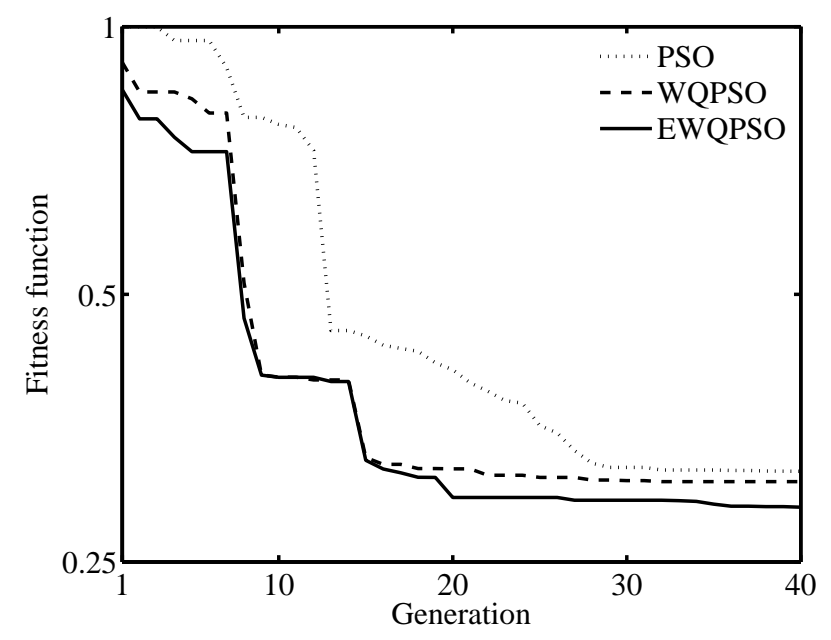

Figure 5: Convergence rate of the EWQPSO, WQPSO, and the classical PSO when applied to the synthesis of the lens antenna shown in Fig. 4. The same number of iterations and population are adopted for the considered algorithms. 


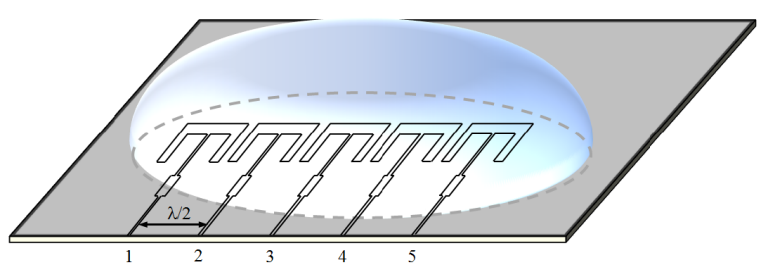

Figure 6: Sketch of the linear patch antenna array used as feeding structure of a supershaped antenna for $60 \mathrm{GHz}$ wireless communications.

\section{Numerical example}

The design of a lens antenna with electronic beam-steering capability is detailed in this section. As shown in Fig. 6, the feeding of the radiating structure is carried out by means of a linear array of five patch antennas optimized in such a way as to achieve good returnloss characteristics in combination with a reasonably small coupling level at the working frequency $f=60 \mathrm{GHz}$. The geometry of the patch array is also illustrated in Fig. 6. The dielectric substrate has a refractive index of 1.8 , whereas the antenna element spacing is set to be $\lambda / 2$, with $\lambda$ denoting the working wavelength in the dielectric lens. As shown in Fig. 7, the radiation pattern of the basic array structure can be electronically steered by changing the phase shift $\Delta \phi$ between adjacent patches. It has been found out that the array directivity ranges from $10.7 \mathrm{dBi}$ for $\Delta \phi=0$ to $11.0 \mathrm{dBi}$ for $\Delta \phi=72^{\circ}$ with a side-lobe level (SLL) of $-9 \mathrm{dBi}$ at the maximum steering angle of $40^{\circ}$. The antenna performance in term of directivity, steering angle and SLL can be enhanced by integrating a suitable dielectric lens (see Fig. 5), whose geometry has been optimized by means of the EWQPSO technique presented in Section IV. To this end, a swarm of $M=24$ particles has been used for the optimization procedure carried out over $g_{\max }=40$ generations. The search domain has been restricted by assuming that the lens parameters $n_{1}, n_{2}, m_{1}, m_{2}, n_{3}, n_{4}, m_{3}, m_{4}, b_{1}, b_{2}$ can range from 1 to 5 , whereas the parameters $a_{1}, a_{2}, a_{3}, a_{4}$ are all set to 1 . Furthermore, the refractive index $n_{d}$ of the material forming the lens can vary between 1.7 to 3 and, finally, the minimal lens radius $r_{\min }$ is assumed to range from $20 \mathrm{~mm}$ to $30 \mathrm{~mm}$. Under these 


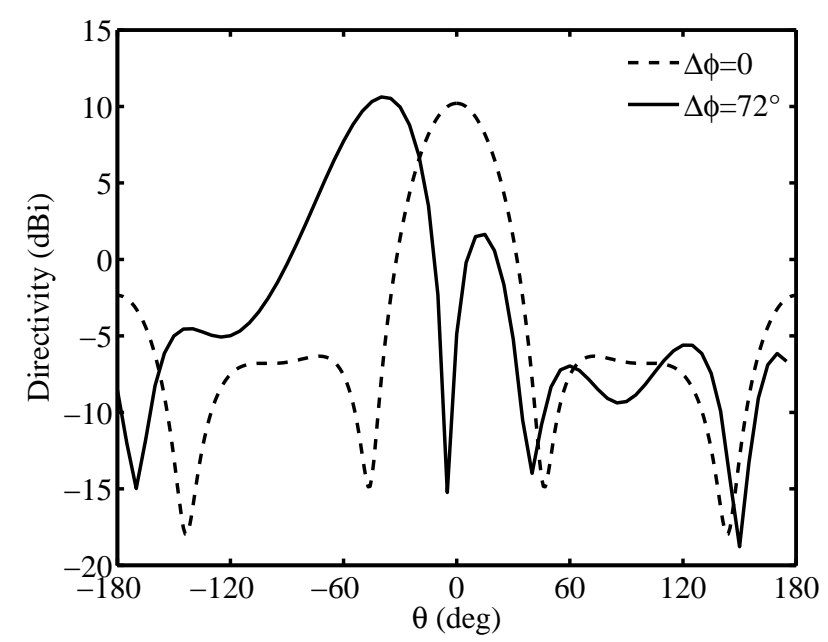

Figure 7: Radiation patterns of the patch antenna array without dielectric lens for different beam-steering angles. Working frequency $f=60 \mathrm{GHz}$.

assumptions, by using the EWQPSO procedure, the optimal lens parameters are found to be: $n_{d}=1.76, r_{\min }=28.4 \mathrm{~mm}, n_{1}=4.341, n_{2}=4.054, m_{1}=1.902, m_{2}=2.072, n_{3}=3.896$, $n_{4}=4.008, m_{3}=1.875, m_{4}=1.947, b_{1}=2.398, b_{2}=2.161$ with the feeding patch array being centered in the origin of the coordinate system. The resulting lens geometry is shown in Fig. 8. The rotational asymmetry of the structure can be noticed. In Fig. 9 the $\mathrm{H}_{-}^{-}$ plane radiation pattern of the array for the excitation phase shifts $\Delta \phi=0, \Delta \phi=72^{\circ}$ and $\Delta \phi=144^{\circ}$ is shown. As it can be noticed in Fig. 9, the considered antenna structure is characterized by a SLL of $-10 \mathrm{dBi}$ for scanning angles up to $50^{\circ}$. Furthermore, the lens integration allows achieving a larger peak directivitiy value ranging from $18.3 \mathrm{dBi}$ for $\Delta \phi=0$ to $17.2 \mathrm{dBi}$ for $\Delta \phi=144^{\circ}$. It is important to notice in Fig. 9 the good agreement between the results obtained by applying the proposed design approach and those computed by using the commercial electromagnetic solver CST Microwave Studio.

\section{Conclusion}

A novel design procedure for supeshaped dielectric lens antennas has been presented. To this end, a GO-PO formulation is used for modeling the multiple reflection processes occurring 


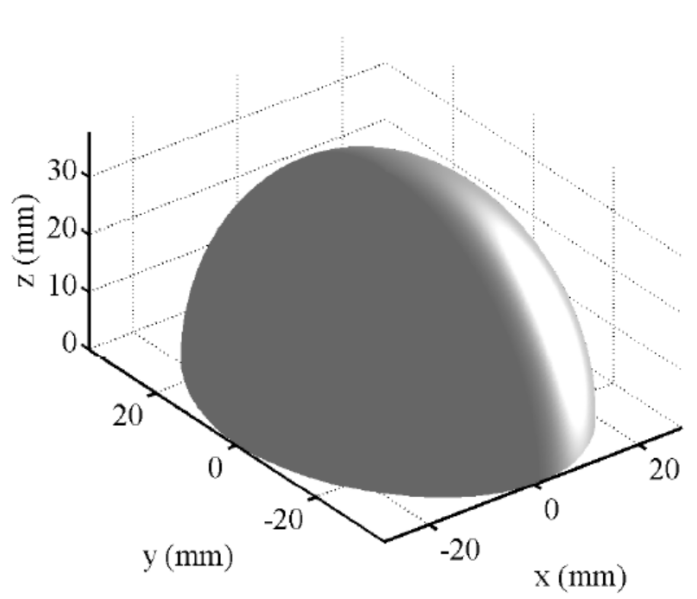

(a)

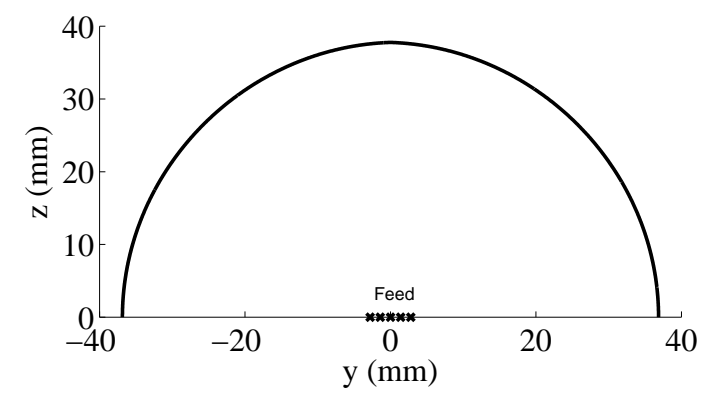

(c)

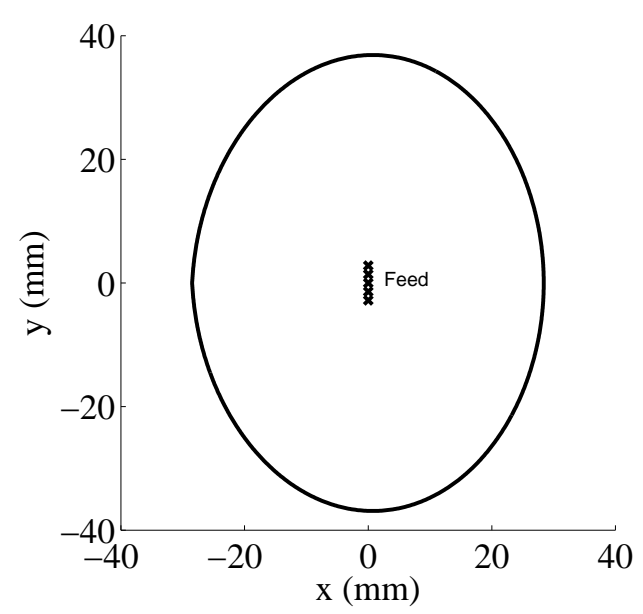

(b)

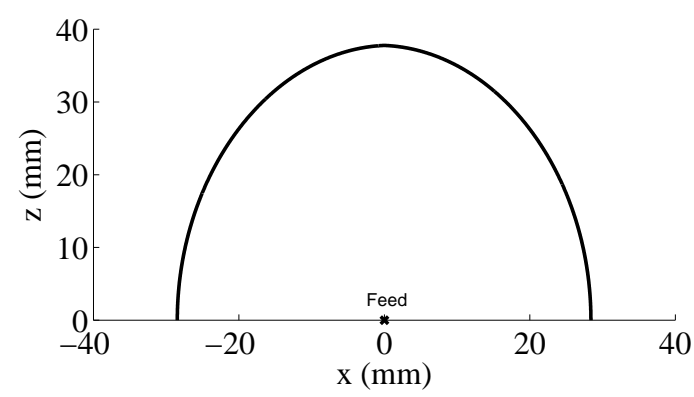

(d)

Figure 8: a) Optimal lens shape synthesized for electronic beam steering, at $60 \mathrm{GHz}$, of the patch antenna array shown in Fig. 6 . b) $\mathrm{z}=0$ cutting plane, c) $\mathrm{x}=0$ cutting plane, d) $\mathrm{y}=0$ cutting plane. 


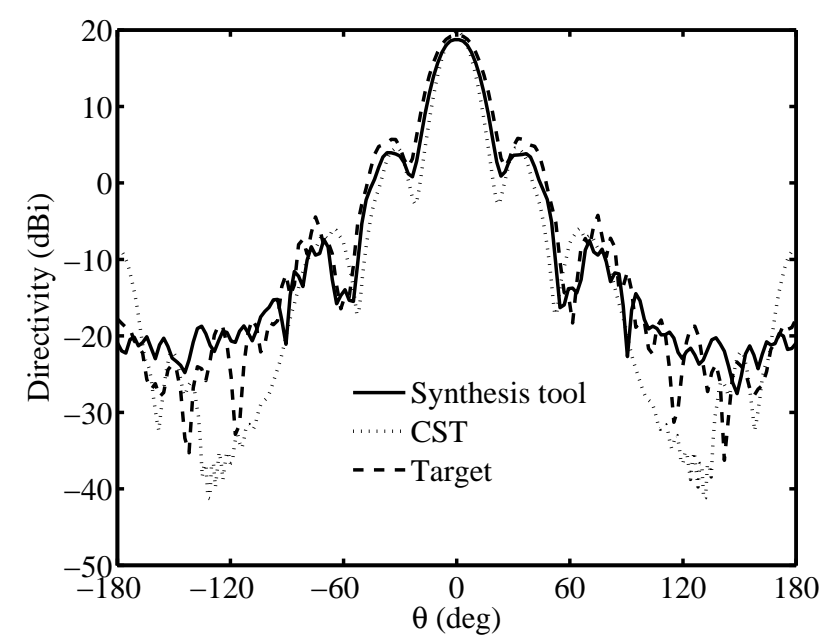

(a)

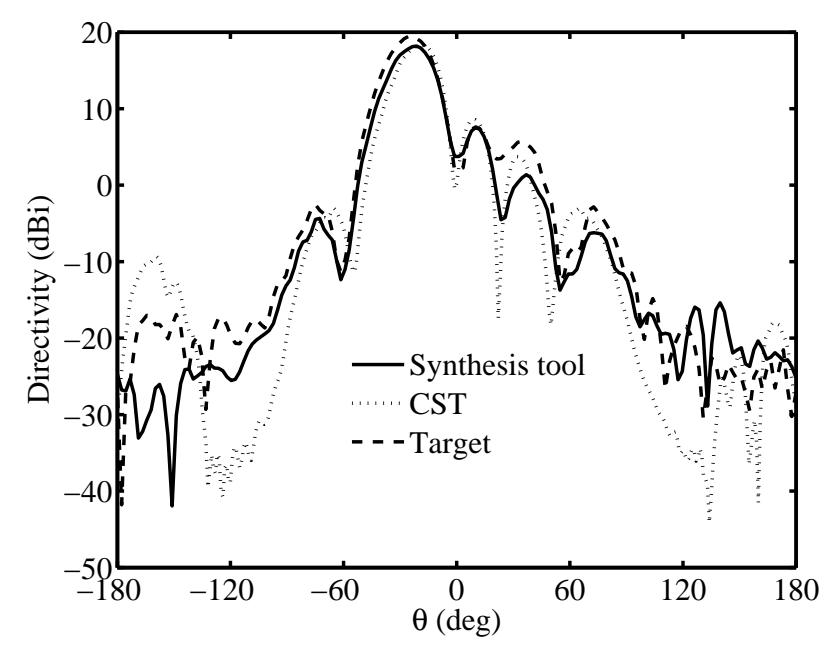

(b)

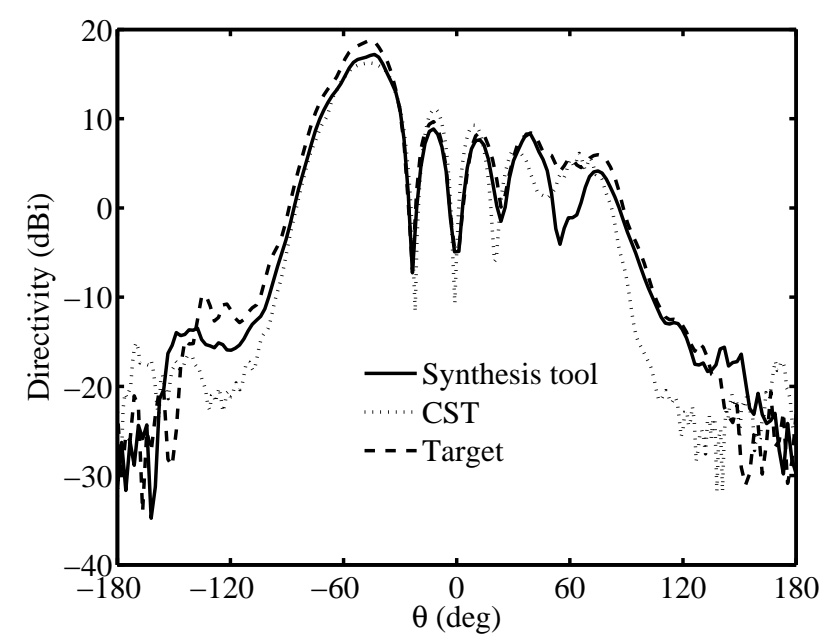

(c)

Figure 9: H-plane radiation pattern of a lens-integrated patch antenna array for different phase excitations a) $\Delta \phi=0$, b) $\Delta \phi=72^{\circ}$, c) $\operatorname{c}$ T Review Copy Only 
within the lens. The proposed model has been validated by comparison with the results obtained by means of the full-wave electromagnetic solver CST Microwave Studio. In order to synthesize lens antennas featuring a given radiation mask, a dedicated EWQPSO optimization procedure has been developed. By using the design tool one can retrieve the optimal lens geometry, size, and material characteristics useful to achieve the desired antenna performance. The applicability of the new optimization procedure was investigated by studying the problem of synthesis of lens antennas having flat-top radiation characteristics as well with electronic beam-steering capability for $60 \mathrm{GHz}$ wireless communications. The performance of the EWQPSO was shown to outperform both the WQPSO and the classical PSO most of the time in the convergence rate as well as the final error level. The obtained results have been found to be in good agreement with those computed by full--wave simulations. The proposed design tool could be usefully adopted to design complex Gielis' lens antennas, for a wide variety of applications ranging from microwave imaging to the $\mathrm{mm}$-wave communications, where enhanced directivity, and complex radiation patterns are needed for optimal radio coverage and illumination, improved efficiency, and reduced electromagnetic interferences between devices sharing the same platform.

\section{Appendix}

In this appendix, the analytical expression of physical quantities used in the GO and PO procedures is provided. The unit vector normal to the surface of the sphere is defined as follows:

$$
\hat{\mathbf{n}}=\frac{\frac{\partial \mathbf{r}}{\partial \theta} \times \frac{\partial \mathbf{r}}{\partial \phi}}{\left\|\frac{\partial \mathbf{r}}{\partial \theta} \times \frac{\partial \mathbf{r}}{\partial \phi}\right\|}
$$


where the derivatives of the radius vector $\mathbf{r}(\theta, \phi)$ are given by:

$$
\begin{aligned}
\frac{\partial \mathbf{r}}{\partial \theta} & =\frac{\partial r}{\partial \theta} \hat{\mathbf{r}}+r \hat{\boldsymbol{\theta}} \\
\frac{\partial \mathbf{r}}{\partial \phi} & =\frac{\partial r}{\partial \phi} \hat{\mathbf{r}}+r \sin \theta \hat{\boldsymbol{\phi}}
\end{aligned}
$$

with

$$
\begin{aligned}
\frac{\partial r}{\partial \theta} & =\frac{\partial r}{\partial \mu} \frac{\partial \mu}{\partial \theta} \\
\frac{\partial r}{\partial \phi} & =\frac{\partial r}{\partial \nu}-\tan \gamma \frac{\partial r}{\partial \theta} \\
\gamma & =\operatorname{atan}\left(\frac{\partial \theta}{\partial \mu}\right)
\end{aligned}
$$

Therefore, upon substituting (29)-(30) in (28) it follows that:

$$
\begin{aligned}
\hat{\mathbf{n}} & =\frac{1}{\left\|\frac{\partial \mathbf{r}}{\partial \theta} \times \frac{\partial \mathbf{r}}{\partial \phi}\right\|}\left|\begin{array}{ccc}
\hat{\mathbf{r}} & \hat{\boldsymbol{\theta}} & \hat{\boldsymbol{\phi}} \\
\frac{\partial r}{\partial \theta} & r & 0 \\
\frac{\partial r}{\partial \phi} & 0 & r \sin \theta
\end{array}\right| \\
& =\frac{r^{2} \sin \theta \hat{\mathbf{r}}-r \sin \theta \frac{\partial r}{\partial \theta} \hat{\boldsymbol{\theta}}-r \frac{\partial r}{\partial \phi} \hat{\boldsymbol{\phi}}}{\left\|\frac{\partial \mathbf{r}}{\partial \theta} \times \frac{\partial \mathbf{r}}{\partial \phi}\right\|}
\end{aligned}
$$

The unit vector tangent to the lens surface $\hat{\mathbf{t}}=t_{r} \hat{\mathbf{r}}+t_{\theta} \hat{\boldsymbol{\theta}}+t_{\phi} \hat{\boldsymbol{\phi}}$ is given by:

$$
\hat{\mathbf{t}}=-\frac{\hat{\mathbf{n}} \times\left(\hat{\mathbf{n}} \times \hat{\mathbf{k}}_{i}\right)}{\left\|\hat{\mathbf{n}} \times\left(\hat{\mathbf{n}} \times \hat{\mathbf{k}}_{i}\right)\right\|}
$$

Upon using the identity $\mathbf{A} \times \mathbf{B} \times \mathbf{C}=\mathbf{B}(\mathbf{A} \cdot \mathbf{C})-\mathbf{C}(\mathbf{A} \cdot \mathbf{B})$ and introducing the incident unit wave vector $\hat{\mathbf{k}}_{i}=k_{i r} \hat{\mathbf{r}}+k_{i \theta} \hat{\boldsymbol{\theta}}+k_{i \phi} \hat{\boldsymbol{\phi}}$ one can obtain the following expression:

$$
\hat{\mathbf{t}}=-\frac{\left[\left(\hat{\mathbf{n}} \cdot \hat{\mathbf{k}}_{i}\right) n_{r}-k_{i r}\right] \hat{\mathbf{r}}+\left[\left(\hat{\mathbf{n}} \cdot \hat{\mathbf{k}}_{i}\right) n_{\theta}-k_{i \theta}\right] \hat{\boldsymbol{\theta}}+\left[\left(\hat{\mathbf{n}} \cdot \hat{\mathbf{k}}_{i}\right) n_{\phi}-k_{i \phi}\right] \hat{\boldsymbol{\phi}}}{\left\|\hat{\mathbf{n}} \times\left(\hat{\mathbf{n}} \times \hat{\mathbf{k}}_{i}\right)\right\|}
$$

In this way the incidence and transmission angles $\theta_{i}$ and $\theta_{t}$ respectively, can be computed as:

$$
\begin{gathered}
\theta_{i}=\tan ^{-1}\left(\frac{\hat{\mathbf{k}}_{i} \cdot \hat{\mathbf{t}}}{\hat{\mathbf{k}}_{i} \cdot \hat{\mathbf{n}}}\right) \\
\theta_{t}=\sin ^{-1}\left(n_{d} \sin \theta_{i}\right) \\
\text { IET Review Copy Only } \\
21
\end{gathered}
$$


The transmitted unit wave vector $\hat{\mathbf{k}}_{t}$ is:

$$
\begin{aligned}
& \hat{\mathbf{k}}_{t}=\cos \theta_{t} \hat{\mathbf{n}}+\sin \theta_{t} \hat{\mathbf{t}}= \\
&\left(\cos \theta_{t} n_{r}+\sin \theta_{t} t_{r}\right) \hat{\mathbf{r}}+\left(\cos \theta_{t} n_{\theta}+\sin \theta_{t} t_{\theta}\right) \hat{\boldsymbol{\theta}}+ \\
&\left(\cos \theta_{t} n_{\phi}+\sin \theta_{t} t_{\phi}\right) \hat{\boldsymbol{\phi}}
\end{aligned}
$$

In order to evaluate the antenna directivity, $h(\alpha, \beta)$, by means of the GO approach, the term $\frac{\partial \alpha}{\partial \theta} \frac{\partial \beta}{\partial \phi}-\frac{\partial \alpha}{\partial \phi} \frac{\partial \beta}{\partial \theta}$ has to be calculated. The angle $\alpha$ is defined as follows:

$$
\alpha=\cos ^{-1}\left(k_{t z}\right)
$$

so that the first derivative with respect to $\theta$ is found to be:

$$
\frac{\partial \alpha}{\partial \theta}=-\frac{1}{\sqrt{1-k_{t z}^{2}}} \frac{\partial k_{t z}}{\partial \theta}
$$

where $k_{t z}$ is the component along the $z$ direction of the transmitted wave vector $\hat{\mathbf{k}}_{t}$ that is:

$$
\left[\begin{array}{l}
k_{t x} \\
k_{t y} \\
k_{t z}
\end{array}\right]=\left[\begin{array}{ccc}
\sin \theta \cos \phi & \cos \theta \cos \phi & -\sin \phi \\
\sin \theta \sin \phi & \cos \theta \sin \phi & \cos \phi \\
\cos \theta & -\sin \theta & 0
\end{array}\right]\left[\begin{array}{c}
k_{t r} \\
k_{t \theta} \\
k_{t \phi}
\end{array}\right]
$$

Furthermore, it is straightforward to verify that:

$$
\frac{\partial \alpha}{\partial \phi}=-\frac{1}{\sqrt{1-k_{t z}^{2}}} \frac{\partial k_{t z}}{\partial \phi}
$$

The angle $\beta$ is defined as follows:

$$
\beta=\tan ^{-1}\left(\frac{k_{t y}}{k_{t x}}\right)
$$

so that:

$$
\begin{gathered}
\frac{\partial \beta}{\partial \theta}=\frac{1}{1-\left(\frac{k_{t y}}{k_{t x}}\right)^{2}}\left(\frac{k_{t x} \frac{\partial k_{t y}}{\partial \theta}-k_{t y} \frac{\partial k_{t x}}{\partial \theta}}{k_{t x}^{2}}\right) \\
\frac{\partial \beta}{\partial \phi}=\frac{1}{1-\left(\frac{k_{t y}}{k_{t x}}\right)^{2}}\left(\frac{k_{t x} \frac{\partial k_{t y}}{\partial \phi}-k_{t y} \frac{\partial k_{t x}}{\partial \phi}}{k_{t x}^{2}}\right) \\
\text { IET Review Copy Only } \\
22
\end{gathered}
$$


Finally, upon setting $\hat{\mathbf{k}}_{t}=k_{t r} \hat{\mathbf{r}}+k_{t \theta} \hat{\boldsymbol{\theta}}+k_{t \phi} \hat{\boldsymbol{\phi}}$, the following expressions can be derived:

$$
\begin{gathered}
\frac{\partial k_{t z}}{\partial \theta}=-\sin \theta\left(k_{t r}+\frac{\partial k_{t \theta}}{\partial \theta}\right)+\cos \theta\left(\frac{\partial k_{t r}}{\partial \theta}-k_{t \theta}\right) \\
\frac{\partial k_{t x}}{\partial \theta}=k_{t r} \cos \theta \sin \phi+\sin \theta \sin \phi \frac{\partial k_{t r}}{\partial \theta}-k_{t \theta} \sin \theta \sin \phi+ \\
\cos \theta \sin \phi \frac{\partial k_{t \theta}}{\partial \theta}+\cos \phi \frac{\partial k_{t \phi}}{\partial \theta} \\
\frac{\partial k_{t y}}{\partial \theta}=k_{t r} \cos \theta \cos \phi+\sin \theta \cos \phi \frac{\partial k_{t r}}{\partial \theta}-k_{t \theta} \sin \theta \cos \phi+ \\
\frac{\partial k_{t z}}{\partial \phi}=\cos \theta \frac{\partial k_{t r}}{\partial \phi}-\sin \theta \frac{\partial k_{t \phi}}{\partial \phi} \\
\frac{\partial k_{t x}}{\partial \phi}=-k_{t r} \sin \theta \sin \phi+\sin \theta \cos \phi \frac{\partial k_{t r}}{\partial \phi}-k_{t \theta} \cos \theta \sin \phi+ \\
\frac{\partial k_{t y}}{\partial \phi}=k_{t r} \sin \theta \cos \phi+\sin \theta \sin \phi \frac{\partial k_{t \theta}}{\partial \phi}-\sin \phi \frac{\partial k_{t \phi}}{\partial \theta} \\
\cos \theta \cos \phi \frac{\partial k_{t \theta}}{\partial \phi}-\sin \phi \frac{\partial k_{t \phi}}{\partial \phi}-\cos \phi k_{t \phi}
\end{gathered}
$$

\section{References}

[1] K. Uehara, K. Miyashita, K. Natsuma, K. Hatakeyama, and K. Mizuno, "Lens-coupled imaging arrays for the millimeter- and submillimeterwave regions," IEEE Trans. Microw. Theory Tech, vol. 40, pp. 806-811, 1992.

[2] S. Raman and N. S. Barker, "Aw-band dielectric-lens-based integrated monopulse radar receiver," IEEE Trans. Microw. Theory Tech., vol. 46, pp. 2308-2316, 1998.

[3] T. H. Bttgenbach, "An improved solution for integrated array optics in quasioptical mm and submm receivers: The hybrid antenna," IEEE Trans. Microw. Theory Tech., vol. 41, pp. 1750-1761, 1998. 
[4] A. Rolland, R. Sauleau, and L. L. Coq, "Flat-shaped dielectric lens antenna for 60-ghz applications," IEEE Trans. Antennas Propagat., vol. 59, pp. 4041-4048, 2011.

[5] D. F. Filipovic and G. M. Rebeiz, "Double-slot antennas on extended hemispherical and elliptical quartz dielectric lenses," Int. J. Infrared Millimeter Waves, vol. 14, pp. 1905-1924, 1993.

[6] J. J. Lee, Handbook of Microwave and Optical Components. New York: K. Chang Ed. Wiley, 1989.

[7] B. Chantraine-Bars, R. Sauleau, and L. L. Coq, "A new accurrate design method for millimeter-wave homogeneous dielectric substrate lens antennas," IEEE Trans. Antennas Propagat., vol. 53, pp. 1069-1082, 2005.

[8] T. Dang, J. Yang, and H. Zheng, "An integrated lens antenna design with irregular lens profile," 5th Global Symposium on Millimeter Waves, pp. 212-215, 2012.

[9] A. Boriskin, G. Godi, R. Sauleau, and A. Nosich, "Small hemielliptic dielectric lens antenna analysis in 2-d: boundary integral equations versus geometrical and physical optics," IEEE Trans. Antennas Propag., vol. 56, pp. 485-492, 2008.

[10] A. P. Pavacic, D. L. del Ro, J. R. Mosig, and G. V. Eleftheriades, "Three-dimensional ray-tracing to model internal reflecition in off-axis lens antennas," IEEE Trans. Antennas Propagat., vol. 54, pp. 604-612, 2006.

[11] A. Neto, S. Maci, and P. J. I. de Maagt, "Reflections inside an elliptical dielectric lens antenna," IEE Proc. Microwaves, Antennas Propagat., vol. 145, 1998.

[12] J. Gielis, "A generic geometric transformation that unifies a wide range of natural and abstract shapes," Amer. J. Botany, vol. 90, pp. 333-338, 2003. 
[13] M. Simeoni, R. Cicchetti, A. Yarovoy, and D. Caratelli, "Plastic-based supershaped dielectric resonator antennas for wide-band applications," IEEE Trans. Antennas Propagat., vol. 59, pp. 4820-4825, 2011.

[14] C. A. Balanis, Antenna Theory: Analysis and Design. Hoboken: 3rd Edition John Wiley and Sons, 2005.

[15] G. Fornarelli and L. Mescia, Swarm intelligence for electric and electronic engineering. Hershey PA: IGI Global, 2013.

[16] Y. T. Lo and V. S. W. Lee, Antenna Handbook. New York USA: vol 2. Van Nostrand Reinhold, 1993.

[17] P. Bia, D. Caratelli, L. Mescia, and J. Gielis, "Electromagnetic characterization of supershaped lens antennas for high-frequency applications," Proceedings of the 43rd European Microwave Conference, pp. 1679-1682, 2013.

[18] M. Xi, J. Sun, and W. Xu, "An improved quantum-behaved particle swarm optimization algorithm with weighted mean best position," Applied Mathmatics and Computation, vol. 205, pp. 751-759, 2012.

[19] N. T. Nguyen, A. Rolland, and R. Sauleau, "Range of validity and accuracy of the hybrid GO-PO method for the analysis of reduced-size lens antennas: benchmarking with BoR-FDTD," Asia-Pacific Microwave Conference, pp. 1-4, 2008. 